\title{
Research Article \\ Elliptic Travelling Wave Solutions to a Generalized Boussinesq Equation
}

\author{
Abdelfattah El Achab \\ Department of Mathematics, Faculty of Sciences, University of Chouaïb Doukkali, BP 20, El-Jadida, Morocco \\ Correspondence should be addressed to Abdelfattah El Achab; abdelfattahelachab@gmail.com
}

Received 6 October 2013; Accepted 2 January 2014; Published 17 February 2014

Academic Editor: Chaudry Masood Khalique

Copyright (C) 2014 Abdelfattah El Achab. This is an open access article distributed under the Creative Commons Attribution License, which permits unrestricted use, distribution, and reproduction in any medium, provided the original work is properly cited.

Travelling wave solutions for the generalized Boussinesq wave equation are studied by using the Weierstrass elliptic function method. As a result, some previously known solutions are recovered, and at the same time some new ones are also given, as well as integrable ones.

\section{Introduction}

It is well known that investigating the exact travelling wave solutions to nonlinear evolution equations plays an important role in the study of nonlinear physical phenomena. In order to obtain the exact solutions, a number of methods have been proposed, such as the homogeneous balance method [1], the hyperbolic function expansion method [2], Jacobi elliptic function method [3], F-expansion method [4], homotopy analysis method $[5,6]$, the bifurcation theory method of dynamical systems $[7,8]$, and Weierstrass elliptic function method [9]. Among these methods, Weierstrass elliptic function method is a powerful mathematic tool to solve nonlinear evolution equations. By using this method, many kinds of important nonlinear evolution equations have been solved successfully $[10,11]$.

To understand the role of nonlinear dispersion in the formation of patterns in liquid drops, Rosenau and Hyman [12] introduced and studied a family of fully nonlinear $K d V$ equations $K(m, n)$ :

$$
u_{t}+\left(u^{m}\right)_{x}+\left(u^{n}\right)_{x x x}=0, \quad m>0,1<n \leq 3 .
$$

A class of solitary waves (which they named compactons) with the special property that, after colliding with other compacton solutions, they reemerge with the same coherent shape was presented. From then on, compacton solutions attracted a lot of interest [13-15]. You can refer to [16-18], for example, for more details about the properties of compacton solutions. Compacton solutions and solitary solutions of other nonlinear evolution equations such as Boussinesq equations and Boussinesq-like $B(m, n)$ equations had been extensively investigated by many authors [19-21]. Recently, Yan [19] introduced a class of fully Boussinesq equations $B(m$, n)

$$
u_{t t}=\left(u^{m}\right)_{x x}+\left(u^{n}\right)_{x x x x}=0, \quad m, n \in \mathbb{R}
$$

and presented some of its compacton solutions when $m=$ $n$. Liu et al. [22] utilized the integral approach to investigate its compacton solutions. More recently, Zhu [20, 21] studied Boussinesq-like $B(m, n)$ equations

$$
\begin{aligned}
& u_{t t}+\left(u^{m}\right)_{x x}-\left(u^{n}\right)_{x x x x}=0, \quad m, n>1, \\
& u_{t t}-\left(u^{m}\right)_{x x}+\left(u^{n}\right)_{x x x x}=0, \quad m, n>1, \\
& u_{t t}+\left(u^{2 n}\right)_{x x}+\left(u^{2 n}\right)_{x x x x}=0, \quad n \geq 1,
\end{aligned}
$$

by using the extended decomposition method.

By making use of the sine-cosine method technique, Wazwaz [23] obtained various forms of travelling wave solutions for improved Boussinesq equations with positive or negative exponents. It was highlighted in [23] that the variants and exponents in the equations directly lead to a qualitative change in the physical structures of the obtained solutions. Lai and $\mathrm{Wu}[24,25]$ proposed an approach for 
constructing asymptotic solutions in a Sobolev space for generalized Boussinesq equations. Using the tanh methods, Wazwaz [26] obtained the compacton solutions, solitons, solitary pattern solutions, and periodic solutions for the Boussinesq wave equation

$$
u_{t t}-u_{x x}+3\left(u^{2}\right)_{x x}+a_{1} u_{x x x x}
$$

and its generalized form

$$
u_{t t}-u_{x x}+\frac{3}{2}\left(u^{m}\right)_{x x}+a_{1} u_{x x x x}
$$

where $a_{1}$ and $m \neq \pm 1$ are constants.

In this paper, we will consider the travelling wave solutions of the following generalized Boussinesq equations ( simply called $\mathrm{GB}(m, n)$ equation):

$$
u_{t t}-u_{x x}+a\left(u^{m}\right)_{x x}+b\left(u^{n}\right)_{x x x x} .
$$

The objective of this paper is to investigate the travelling wave solutions of (6) systematically, by applying the Weierstrass elliptic function method. It will be shown that some previously known solutions are recovered, and at the same time, some new ones are also given.

The rest of this paper is organized as follows. In Section 2, we first outline the Weierstrass elliptic function method which will be used in the next section. In Section 3, we give some particular travelling wave solutions of (6). Finally, some conclusions are given in Section 4.

\section{Weierstrass Elliptic Functions}

Let us consider the following nonlinear differential equation:

$$
\left(\frac{d \phi(z)}{d z}\right)^{2}=a_{0} \phi^{4}+4 a_{1} \phi^{3}+6 a_{2} \phi^{2}+4 a_{3} \phi+a_{4} \equiv f(\phi) \text {. }
$$

As is well known $[27,28]$, the solutions $\phi$ of (7) can be expressed in terms of elliptic functions $\wp$. It reads as

$$
\phi(z)=\phi_{0}+\frac{f^{\prime}\left(\phi_{0}\right)}{4\left[\wp\left(z, g_{2}, g_{3}\right)-(1 / 24) f^{\prime \prime}\left(\phi_{0}\right)\right]},
$$

where the primes denote differentiation with respect to $\phi$ and $\phi_{0}$ is a simple root of $f(\phi)$. The invariants $g_{2}, g_{3}$ of elliptic functions $\wp\left(t, g_{2}, g_{3}\right)$ are related to the coefficients of $f(\phi)$ by [29]

$$
\begin{gathered}
g_{2}=a_{0} a_{4}-4 a_{1} a_{3}+3 a_{2}^{2}, \\
g_{3}=a_{0} a_{2} a_{4}+2 a_{1} a_{2} a_{3}-a_{2}^{3}-a_{0} a_{3}^{2}-a_{1}^{2} a_{4} .
\end{gathered}
$$

When $g_{2}$ and $g_{3}$ are real and the discriminant

$$
\Delta=g_{2}^{3}-27 g_{3}^{2}
$$

is positive, negative, or zero, we have different behavior of $\wp(t)$. The conditions [9]

$$
\Delta \neq 0 \quad \text { or } \quad \Delta=0, \quad g_{2}>0, \quad g_{3}>0
$$

lead to periodic solutions, whereas the conditions

$$
\Delta=0, \quad g_{2} \geq 0, \quad g_{3} \leq 0
$$

lead to solitary wave solutions.

If $\Delta=0$, then $\wp\left(t, g_{2}, g_{3}\right)$ degenerates into trigonometric or hyperbolic functions [30]. Thus, periodic solutions according to (8) are determined by

$$
\begin{array}{r}
\phi(z)=\phi_{0} \\
+f^{\prime}\left(\phi_{0}\right) \times\left(4 \left[-\frac{e_{1}}{2}-\frac{f^{\prime \prime}\left(\phi_{0}\right)}{24}\right.\right. \\
\left.\left.+\frac{3}{2} e_{1} \csc ^{2}\left(\sqrt{\frac{3}{2} e_{1} t}\right)\right]\right)^{-1}, \\
\Delta=0, g_{3}>0,
\end{array}
$$

and solitary wave solutions by

$$
\begin{aligned}
& \phi(z)=\phi_{0} \\
& +f^{\prime}\left(\phi_{0}\right) \times\left(4 \left[e_{1}-\frac{f^{\prime \prime}\left(\phi_{0}\right)}{24}\right.\right. \\
& \left.\left.+3 e_{1} \operatorname{csch}^{2}\left(\sqrt{3 e_{1}} t\right)\right]\right)^{-1}, \\
& \Delta=0, g_{3}<0,
\end{aligned}
$$

where $e_{1}=\sqrt[3]{\left|g_{3}\right|}$ in (13) and $e_{1}=(1 / 2) \sqrt[3]{\left|g_{3}\right|}$ in (14).

\section{Travelling Wave Solutions of the Generalized Boussinesq Equations}

In this section, we consider the travelling wave solutions of (6). Assume that (6) has an exact solution in the form of a travelling wave

$$
u(x, t)=u(\xi), \quad \xi=\mu\left(x-c t-x_{0}\right)
$$

where $\mu \neq 0, c \neq 0$, and $x_{0}$ are arbitrary constants. Substituting (15) into (6), we get

$$
\left(c^{2}-1\right) u^{\prime \prime}+a\left(u^{m}\right)^{\prime \prime}+b \mu^{2}\left(u^{n}\right)^{\prime \prime \prime \prime} .
$$

Integrating (16) twice and letting the constants of integration be zero give rise to

$$
\left(c^{2}-1\right) u+a\left(u^{m}\right)+b \mu^{2}\left(u^{n}\right)^{\prime \prime}=0 .
$$

Making transformation, $u^{n}=\varphi$, and (17) becomes

$$
\left(c^{2}-1\right) \varphi^{1 / n}+a\left(\varphi^{m / n}\right)+b \mu^{2} \varphi^{\prime \prime}=0 .
$$


Multiply (18) by the integrating factor $\varphi^{\prime}$ on both sides, and integrating it with respect to $\xi$, we have

$$
\left(\frac{d \varphi}{d \xi}\right)^{2}=\frac{2}{b \mu^{2}}\left[C_{1}-\frac{n\left(c^{2}-1\right)}{n+1} \varphi^{1+(1 / n)}-\frac{a n}{m+n} \varphi^{1+(m / n)}\right]
$$

where $C_{1}$ is an integration constant.

Making the transformation $\varphi=\phi^{p}, p \neq 0$ we get

$$
\begin{aligned}
\left(\frac{d \phi}{d \xi}\right)^{2}= & \frac{2}{b \mu^{2} p^{2}} \\
& \times\left[C_{1} \phi^{2-2 p}-\frac{n\left(c^{2}-1\right)}{n+1} \phi^{2+((1 / n)-1) p}\right. \\
& \left.\quad-\frac{a n}{m+n} \phi^{2+((m / n)-1) p}\right] .
\end{aligned}
$$

In order to guarantee the integrability of (20), the powers of $\phi$ have to be integer numbers between 0 and 4 , and therefore we have the following parameter conditions:

(1) if $C_{1}=0, n=1$, then $p \in\{-2 / m,-1 /(m-1), 2 /(m-$ $1), 1 /(m-1)\}$;

(2) if $C_{1}=0, n \neq 1, n=m+1$, then $p \in\{m /(m-$ $1),-m /(m-1), 2 m /(m-1),-2 m /(m-1)\}$;

(3) if $C_{1} \neq 0, n=1$, then we have $m=5$ and $p= \pm 1 / 2$;

(4) if $n=p=1$, then we have $m=2$ or $m=3$.

Next, using the basic results on Weierstrass elliptic functions shown in Section 2, we will analyze the solutions of (20) in the above cases.

3.1. Case 1. (i) If $C_{1}=0, n=1$, and $p=1 /(m-1)$, (20) becomes

$$
\left(\frac{d \phi}{d \xi}\right)^{2}=\frac{2(m-1)^{2}}{b \mu^{2}}\left[\frac{\left(1-c^{2}\right)}{2} \phi^{2}-\frac{a}{m+1} \phi^{3}\right]=f(\phi) .
$$

It is easy to see that $f(\phi)$ has two roots: $\phi_{0}=0$ and $\phi_{0}=$ $\left(\left(1-c^{2}\right)(m+1)\right) / 2 a$. From (8), we can obtain the following solution for (21):

$$
\phi=\phi_{0}+\frac{6(m-1)\left(\left(1-c^{2}\right) \phi_{0}-(3 a /(m+1)) \phi_{0}^{2}\right)}{12 b \mu^{2} \wp(\xi)-(m-1)\left(1-c^{2}-(6 a /(m+1))\right)},
$$

where the invariants are

$$
g_{2}=\frac{(m-1)^{4}\left(1-c^{2}\right)^{2}}{12\left(b \mu^{2}\right)^{2}}, \quad g_{3}=-\frac{(m-1)^{6}\left(1-c^{2}\right)^{3}}{216\left(b \mu^{2}\right)^{3}} .
$$

Then the discriminant $\Delta=0$. The root $\phi_{0}=0$ gives the trivial solution $\phi=0$, and the nonzero solution of (21) can be found by taking $\phi_{0}=\left(\left(1-c^{2}\right)(m+1)\right) / 2 a$. Hence, from (11), we have the periodic wave solution to (21)

$$
\phi=\frac{\left(1-c^{2}\right)(m+1)}{2 a} \sec ^{2}\left(\frac{1}{2} \sqrt{-\frac{(m-1)^{2}\left(1-c^{2}\right)}{\left(b \mu^{2}\right)}} \xi\right)
$$

for $\left(1-c^{2}\right) / b<0$. From (12), we have the solitary wave solution

$$
\phi=\frac{\left(1-c^{2}\right)(m+1)}{2 a} \operatorname{sech}^{2}\left(\frac{1}{2} \sqrt{\frac{(m-1)^{2}\left(1-c^{2}\right)}{\left(b \mu^{2}\right)}} \xi\right)
$$

for $\left(1-c^{2}\right) / b>0$.

Therefore, when $\left(1-c^{2}\right) / b<0, \operatorname{GB}(m, 1)$ (6) has the following periodic wave solution:

$$
\begin{aligned}
& u(x, t) \\
& =\left(\frac{\left(1-c^{2}\right)(m+1)}{2 a}\right. \\
& \left.\quad \times \sec ^{2}\left(\frac{1}{2} \sqrt{-\frac{(m-1)^{2}\left(1-c^{2}\right)}{\left(b \mu^{2}\right)} \xi}\right)\right)^{1 /(m-1)} .
\end{aligned}
$$

When $\left(1-c^{2}\right) / b<0, \mathrm{~GB}(m, 1)(6)$ has the following solitary wave solution:

$$
\begin{aligned}
& u(x, t) \\
& =\left(\frac{\left(1-c^{2}\right)(m+1)}{2 a}\right. \\
& \left.\quad \times \operatorname{sech}^{2}\left(\frac{1}{2} \sqrt{\frac{(m-1)^{2}\left(1-c^{2}\right)}{\left(b \mu^{2}\right)}} \xi\right)\right)^{1 /(m-1)} .
\end{aligned}
$$

Remark 1. When $m>1$, the solutions (26)-(27) that we obtained are completely the same as (22)-(24) that were obtained by Lai [31].

(ii) If $C_{1}=0, n=1$, and $p=-1 /(m-1),(20)$ becomes

$$
\left(\frac{d \phi}{d \xi}\right)^{2}=\frac{2(m-1)^{2}}{b \mu^{2}}\left[\frac{\left(1-c^{2}\right)}{2} \phi^{2}-\frac{a}{m+1} \phi\right]=f(\phi) .
$$


It is easy to see that $f(\phi)$ has two roots: $\phi_{0}=0$ and $\phi_{0}=2 a /\left(\left(1-c^{2}\right)(m+1)\right)$. From (8), we can obtain that the expression for the solutions of (28) is

$$
\begin{aligned}
\phi= & \left(12 b \mu^{2} \phi_{0} \wp(\xi)+5(m-1)^{2}\left(1-c^{2}\right) \phi_{0}\right. \\
& \left.-\frac{6 a(m-1)^{2}}{(m+1)}\right) \\
& \times\left(12 b \mu^{2} \wp(\xi)-(m-1)^{2}\left(1-c^{2}\right)\right)^{-1},
\end{aligned}
$$

where the invariants are given by (23). Taking the $\operatorname{root} \phi_{0}=0$ into (29), we get

$$
\phi=\frac{-6 a(m-1)^{2}}{(m+1)\left[12 b \mu^{2} \wp(\xi)-(m-1)^{2}\left(1-c^{2}\right)\right]} .
$$

Since the discriminant $\Delta=0$, thus from (11), we have the periodic wave solution to (28)

$$
\phi=\frac{2 a}{\left(1-c^{2}\right)(m+1)} \sin ^{2}\left(\frac{1}{2} \sqrt{-\frac{(m-1)^{2}\left(1-c^{2}\right)}{b \mu^{2}} \xi}\right),
$$

for $\left(1-c^{2}\right) / b<0$. From (12), we have the solitary wave solution

$$
\phi=-\frac{2 a}{\left(1-c^{2}\right)(m+1)} \sinh ^{2}\left(\frac{1}{2} \sqrt{\frac{(m-1)^{2}\left(1-c^{2}\right)}{b \mu^{2}}} \xi\right)
$$

for $\left(1-c^{2}\right) / b>0$.

Therefore, when $\left(1-c^{2}\right) / b<0$, the $\operatorname{GB}(m, 1)(6)$ has the following periodic wave solution:

$$
\begin{aligned}
& u(x, t) \\
& =\left(\frac{\left(1-c^{2}\right)(m+1)}{2 a}\right. \\
& \left.\quad \times \csc ^{2}\left(\frac{1}{2} \sqrt{-\frac{(m-1)^{2}\left(1-c^{2}\right)}{\left(b \mu^{2}\right)} \xi}\right)\right)^{1 /(m-1)} .
\end{aligned}
$$

When $\left(1-c^{2}\right) / b>0$, the $\operatorname{GB}(m, 1)(6)$ has the following solitary wave solution:

$$
\begin{aligned}
& u(x, t) \\
& =\left(-\frac{\left(1-c^{2}\right)(m+1)}{2 a}\right. \\
& \left.\quad \times \operatorname{csch}^{2}\left(\frac{1}{2} \sqrt{\frac{(m-1)^{2}\left(1-c^{2}\right)}{\left(b \mu^{2}\right)} \xi}\right)\right)^{1 /(m-1)} .
\end{aligned}
$$

When substituting the second $\operatorname{root} \phi_{0}=2 a /\left(\left(1-c^{2}\right)(m+1)\right)$ into (29), we have

$$
\begin{aligned}
\phi= & \left(24 a b \mu^{2} \wp(\xi)+5(m+1)(m-1)^{2}\left(1-c^{2}\right)^{2}\right. \\
& \left.-6 a(m-1)^{2}\left(1-c^{2}\right)\right) \\
\times & \left((m+1)\left(1-c^{2}\right)\left[12 b \mu^{2} \wp(\xi)-(m-1)^{2}\left(1-c^{2}\right)\right]\right)^{-1} .
\end{aligned}
$$

So from (11), we have the periodic wave solution to (28):

$$
\phi=\frac{2 a}{\left(1-c^{2}\right)(m+1)} \cos ^{2}\left(\frac{1}{2} \sqrt{-\frac{(m-1)^{2}\left(1-c^{2}\right)}{\left(b \mu^{2}\right)} \xi}\right),
$$

for $\left(1-c^{2}\right) / b<0$. From (12), we have the solitary wave solution to (28):

$$
\phi=\frac{2 a}{\left(1-c^{2}\right)(m+1)} \cosh ^{2}\left(\frac{1}{2} \sqrt{\frac{(m-1)^{2}\left(1-c^{2}\right)}{\left(b \mu^{2}\right)}} \xi\right) .
$$

Therefore, when $\left(1-c^{2}\right) / b<0, \mathrm{~GB}(m, 1)(6)$ has the following periodic wave solution:

$$
\begin{aligned}
& u(x, t) \\
& =\left(\frac{2 a}{\left(1-c^{2}\right)(m+1)}\right. \\
& \left.\quad \times \cos ^{2}\left(\frac{1}{2} \sqrt{-\frac{(m-1)^{2}\left(1-c^{2}\right)}{\left(b \mu^{2}\right)} \xi}\right)\right)^{1 /(1-m)} .
\end{aligned}
$$


When $\left(1-c^{2}\right) / b>0$, the $\operatorname{GB}(m, 1)$ (6) has the following solitary wave solution:

$$
\begin{aligned}
& u(x, t) \\
& =\left(\frac{2 a}{\left(1-c^{2}\right)(m+1)}\right. \\
& \left.\quad \times \cosh ^{2}\left(\frac{1}{2} \sqrt{\frac{(m-1)^{2}\left(1-c^{2}\right)}{\left(b \mu^{2}\right)} \xi}\right)\right)^{1 /(1-m)} .
\end{aligned}
$$

Remark 2. When $m>1$, the solutions (33)-(34) that we obtained are completely the same as (23)-(25) that were obtained by Lai [31]; when $m<1$ and $m \neq-1$, the solutions (38)-(39) that we obtained are similar as the solutions (26)(28) that were obtained by Lai [31].

(iii) If $C_{1}=0, n=1$, and $p=2 /(m-1)$, (20) becomes

$$
\left(\frac{d \phi}{d \xi}\right)^{2}=\frac{(m-1)^{2}}{2 b \mu^{2}}\left[\frac{\left(1-c^{2}\right)}{2} \phi^{2}-\frac{a}{m+1} \phi^{4}\right]=f(\phi) \text {. }
$$

According to (8), the solutions of (40) read

$$
\begin{aligned}
& \phi= \phi_{0}+\left(3(m-1)^{2}\left[\left(1-c^{2}\right) \phi_{0}-\frac{4 a}{m+1} \phi_{0}^{3}\right]\right) \\
& \times\left(24 b \mu^{2} \wp(\xi)-(m-1)^{2}\right. \\
&\left.\times\left(\frac{\left(1-c^{2}\right)}{2}-\frac{6 a}{m+1} \phi_{0}^{2}\right)\right)^{-1},
\end{aligned}
$$

where the invariants are

$$
g_{2}=\frac{(m-1)^{4}\left(1-c^{2}\right)^{2}}{192\left(b \mu^{2}\right)^{2}}, \quad g_{3}=-\frac{(m-1)^{6}\left(1-c^{2}\right)^{3}}{\left(24 b \mu^{2}\right)^{3}} .
$$

Taking the root $\phi_{0}=0$ into (41), we get the trivial solution $\phi=$ 0 . However, when taking the root $\phi= \pm \sqrt{\left(1-c^{2}\right)(m+1) / 2 a}$ into (41), we can obtain the solutions to (40)

$$
\phi= \pm \sqrt{\frac{\left(1-c^{2}\right)(m+1)}{2 a}} \frac{48 b \mu^{2} \wp(\xi)-(m-1)^{2}\left(1-c^{2}\right)}{48 b \mu^{2} \wp(\xi)+5(m-1)^{2}\left(1-c^{2}\right)} .
$$

Since the discriminant $\Delta=0$, it is easy to see from (11), (12) that the above solutions (43) will generate the same periodic and solitary wave solutions to (6) as (26), (27).

(iv) If $C_{1}=0, n=1$, and $p=-2 /(m-1),(20)$ becomes

$$
\left(\frac{d \phi}{d \xi}\right)^{2}=\frac{(m-1)^{2}}{2 b \mu^{2}}\left[\frac{\left(1-c^{2}\right)}{2} \phi^{2}-\frac{a}{m+1}\right]=f(\phi) \text {. }
$$

Using the same arguments as the above, we can deduce that it would give exactly the same solutions of (6) as Case 1 (ii).
3.2. Case 2. (i) If $C_{1}=0, n \neq 1, n=m$, and $p=-m /(m-1)$, (20) becomes

$$
\left(\frac{d \phi}{d \xi}\right)^{2}=\frac{2(m-1)^{2}}{b \mu^{2} m^{2}}\left[\frac{m\left(1-c^{2}\right)}{m+1} \phi^{3}-\frac{a}{2} \phi^{2}\right]=f(\phi) .
$$

Using similar arguments to Case 1 (i), we can deduce that when $a / b>0$, the $\operatorname{GB}(m, m)(6)$ has the following periodic wave solution:

$$
u(x, t)=\left(\frac{a(m+1)}{2 m\left(1-c^{2}\right)} \sec ^{2}\left(\frac{1}{2}\left|\frac{m-1}{m}\right| \sqrt{\frac{a}{b \mu^{2}} \xi}\right)\right)^{-1 /(m-1)} .
$$

When $a / b<0$, the $\operatorname{GB}(m, m)(6)$ has the following solitary wave solution:

$$
\begin{aligned}
& u(x, y, t) \\
& \quad=\left[\frac{a(m+1)}{2 m\left(1-c^{2}\right)} \operatorname{sech}^{2}\left(\frac{1}{2}\left|\frac{m-1}{m}\right| \sqrt{-\frac{a}{b \mu^{2}}} \xi\right)\right]^{-1 /(m-1)} .
\end{aligned}
$$

Remark 3. When $m<1$, the solutions (46)-(47) that we obtained are completely the same as (62)-(64) that were obtained by Lai [31].

(ii) If $C_{1}=0, n \neq 1, n=m$, and $p=m /(m-1)$, becomes

$$
\left(\frac{d \phi}{d \xi}\right)^{2}=\frac{2(m-1)^{2}}{b \mu^{2} m^{2}}\left[\frac{m\left(1-c^{2}\right)}{m+1} \phi-\frac{a}{2} \phi^{2}\right]=f(\phi) .
$$

Using similar arguments to Case 1 (ii), we can deduce that when $a / b>0$, the $\operatorname{GB}(m, m)(6)$ has the following periodic wave solution:

$$
u(x, t)=\left[\frac{2 m\left(1-c^{2}\right)}{a(m+1)} \sin ^{2}\left(\frac{1}{2}\left|\frac{m-1}{m}\right| \sqrt{\frac{a}{b \mu^{2}} \xi}\right)\right]^{1 /(m-1)} .
$$

When $a / b<0$, the $\operatorname{GB}(m, m)(6)$ has the following solitary wave solution:

$$
u(x, t)=\left[\frac{2 m\left(1-c^{2}\right)}{a(m+1)} \sinh ^{2}\left(\frac{1}{2}\left|\frac{m-1}{m}\right| \sqrt{\frac{a}{b \mu^{2}} \xi}\right)\right]^{1 /(m-1)} .
$$

Remark 4. When $m>1$, solutions (49)-(50) that we obtained are completely the same as (59)-(61) that were obtained by Lai [31]. becomes

(iii) If $C_{1}=0, n \neq 1, n=m$, and $p=-2 m /(m-1)$,

$$
\left(\frac{d \phi}{d \xi}\right)^{2}=\frac{(m-1)^{2}}{2 b \mu^{2} m^{2}}\left[\frac{m\left(1-c^{2}\right)}{m+1} \phi^{4}-\frac{a}{2} \phi^{2}\right]=f(\phi) .
$$


Using similar arguments to Case 2 (i), we can deduce that it would give exactly the same solutions of the $\operatorname{GB}(m, m)(6)$ as those given by (45) and (46).

(iv) If $C_{1}=0, n \neq 1, n=m$, and $p=2 m /(m-1)$,

$$
\left(\frac{d \phi}{d \xi}\right)^{2}=\frac{(m-1)^{2}}{2 b \mu^{2} m^{2}}\left[\frac{m\left(1-c^{2}\right)}{m+1}-\frac{a}{2} \phi^{2}\right]=f(\phi) .
$$

Using similar arguments to Case 2 (ii), we can deduce that it would give exactly the same solutions of the $\operatorname{GB}(m, m)(6)$ as those given by (48) and (49).

3.3. Case 3. (i) If $C_{1} \neq 0, n=1, m=5$, and $p=1 / 2$, (20) becomes

$$
\left(\frac{d \phi}{d \xi}\right)^{2}=\frac{8}{b \mu^{2}}\left[C_{1} \phi+\frac{\left(1-c^{2}\right)}{2} \phi^{2}-\frac{a}{6} \phi^{4}\right]=f(\phi) .
$$

According to (8), the solutions of (52) read

$$
\phi=\frac{3 b \mu^{2} \phi_{0} \wp(\xi)+5\left(1-c^{2}\right) \phi_{0}-2 a \phi_{0}^{3}+6 C_{1}}{3 b \mu^{2} \wp(\xi)-\left(1-c^{2}\right)+2 a \phi_{0}^{2}},
$$

where the invariants are given by

$$
g_{2}=\frac{4\left(1-c^{2}\right)^{2}}{3\left(b \mu^{2}\right)^{2}}, \quad g_{3}=-\frac{8\left(1-c^{2}\right)^{3}}{27\left(b \mu^{2}\right)^{3}}+\frac{16 a C_{1}^{2}}{3\left(b \mu^{2}\right)^{3}} \text {. }
$$

So we can obtain the general expressions for the solutions to the $\operatorname{GB}(5,1)(6)$ :

$$
u(x, t)=\left[\frac{3 b \mu^{2} \phi_{0} \wp(\xi)+5\left(1-c^{2}\right) \phi_{0}-2 a \phi_{0}^{3}+6 C_{1}}{3 b \mu^{2} \wp(\xi)-\left(1-c^{2}\right)+2 a \phi_{0}^{2}}\right]^{1 / 2}
$$

For example, substituting the simplest root $\phi=0$ of $f(\phi)$ into (56), we get

$$
u(x, t)=\left[\frac{6 C_{1}}{3 b \mu^{2} \wp(\xi)-\left(1-c^{2}\right)}\right]^{1 / 2} .
$$

(ii) If $C_{1} \neq 0, n=1, m=5$, and $p=-1 / 2$, (20) becomes

$$
\left(\frac{d \phi}{d \xi}\right)^{2}=\frac{8}{b \mu^{2}}\left[C_{1} \phi^{3}+\frac{\left(1-c^{2}\right)}{2} \phi^{2}-\frac{a}{6}\right]=f(\phi) .
$$

Using similar arguments to Case 3 (i), we can get the following general expression for the solutions to $\operatorname{GB}(5,1)(6)$ :

$$
u(x, t)=\left[\frac{3 b \mu^{2} \phi_{0} \wp(\xi)+3\left(1-c^{2}\right) \phi_{0}+12 C_{1} \phi_{0}^{2}}{3 b \mu^{2} \wp(\xi)-\left(1-c^{2}\right)-6 C_{1}}\right]^{-1 / 2}
$$

where the invariants are given by (55).
3.4. Case 4. (i) If $n=1, m=2$, and $p=1,(20)$ becomes

$$
\left(\frac{d \phi}{d \xi}\right)^{2}=\frac{2}{b \mu^{2}}\left[C_{1}+\frac{\left(1-c^{2}\right)}{2} \phi^{2}-\frac{a}{3} \phi^{3}\right]=f(\phi)
$$

Following the same procedure as mentioned above, we can get the general solutions to $\operatorname{GB}(2,1)(6)$ :

$$
u(x, t)=\left[\frac{12 b \mu^{2} \phi_{0} \wp(\xi)+5\left(1-c^{2}\right) \phi_{0}-4 a \phi_{0}^{2}}{12 b \mu^{2} \wp(\xi)-\left(1-c^{2}\right)+2 a \phi_{0}}\right],
$$

where $\phi_{0}$ is the real root of $f(\phi)=0$ and the invariants are given by

$$
g_{2}=3 \frac{4\left(1-c^{2}\right)^{2}}{6\left(b \mu^{2}\right)^{2}}, \quad g_{3}=-\frac{\left(1-c^{2}\right)^{3}}{216\left(b \mu^{2}\right)^{3}}-\frac{a^{2} C_{1}}{108\left(b \mu^{2}\right)^{3}} .
$$

(ii) If $n=1, m=3$, and $p=1,(20)$ becomes

$$
\left(\frac{d \phi}{d \xi}\right)^{2}=\frac{2}{b \mu^{2}}\left[C_{1}+\frac{\left(1-c^{2}\right)}{2} \phi^{2}-\frac{a}{4} \phi^{4}\right]=f(\phi) .
$$

Likewise, we can get the general solutions to $\operatorname{GB}(3,1)(6)$ :

$$
u(x, t)=\left[\frac{12 b \mu^{2} \phi_{0} \wp(\xi)+5\left(1-c^{2}\right) \phi_{0}-3 a \phi_{0}^{3}}{12 b \mu^{2} \wp(\xi)-\left(1-c^{2}\right)+3 a \phi_{0}^{2}}\right],
$$

where $\phi_{0}$ is the real root of $f(\phi)=0$.

\section{Conclusion}

From the above discussion, we find the traveling wave solutions of the generalized Boussinesq equation $\operatorname{GB}(m, n)$, which are expressed by the hyperbolic functions and trigonometric functions, out without the aid of mathematical software. The results show that the Weierstrass function method is a powerful mathematical tool to search for exact solutions to nonlinear differential equations, especially solitary ones. It may be advantageous that this quite general method can lead to free parameters as shown in the solution. We believe that this approach can also be used to solve other nonlinear equations.

\section{Conflict of Interests}

The author declares that there is no conflict of interests regarding the publication of this paper.

\section{References}

[1] M. L. Wang, "Solitary wave solutions for variant Boussinesq equations," Physics Letters A, vol. 199, no. 3-4, pp. 169-172, 1995.

[2] E. J. Parkes and B. R. Duffy, "Travelling solitary wave solutions to a compound KdV-Burgers equation," Physics Letters A, vol. 229, no. 4, pp. 217-220, 1997. 
[3] Z. Fu, S. Liu, S. Liu, and Q. Zhao, "New Jacobi elliptic function expansion and new periodic solutions of nonlinear wave equations," Physics Letters A, vol. 290, no. 1-2, pp. 72-76, 2001.

[4] M. Wang and Y. Zhou, "The periodic wave solutions for the Klein-Gordon-Schrödinger equations," Physics Letters A, vol. 318, no. 1-2, pp. 84-92, 2003.

[5] J.-H. He, "Application of homotopy perturbation method to nonlinear wave equations," Chaos, Solitons and Fractals, vol. 26, no. 3, pp. 695-700, 2005.

[6] J.-H. He, "Homotopy perturbation method for bifurcation of nonlinear problems," International Journal of Nonlinear Sciences and Numerical Simulation, vol. 6, no. 2, pp. 207-208, 2005.

[7] J. Li and Z. Liu, "Smooth and non-smooth traveling waves in a nonlinearly dispersive equation," Applied Mathematical Modelling, vol. 25, no. 1, pp. 41-56, 2000.

[8] J. Li and Z. Liu, "Traveling wave solutions for a class of nonlinear dispersive equations," Journal of Chinese Annals of Mathematics B, vol. 23, no. 3, pp. 397-418, 2002.

[9] H. W. Schürmann, "Traveling-wave solutions of the cubicquintic nonlinear Schrödinger equation," Physical Review E, vol. 54, no. 4, pp. 4312-4320, 1996.

[10] H. W. Schürmann, V. S. Serov, and J. Nickel, "Superposition in nonlinear wave and evolution equations," International Journal of Theoretical Physics, vol. 45, no. 6, pp. 1057-1073, 2006.

[11] J. Nickel, V. S. Serov, and H. W. Schürmann, "Some elliptic traveling wave solutions to the Novikov-Veselov equation," Progress in Electromagnetics Research, vol. 61, pp. 323-331, 2006.

[12] P. Rosenau and J. M. Hyman, "Compactons: solitons with finite wavelength," Physical Review Letters, vol. 70, no. 5, pp. 564-567, 1993.

[13] Z. Liu and J. Li, "Bifurcations of solitary waves and domain wall waves for KdV-like equation with higher order nonlinearity," International Journal of Bifurcation and Chaos, vol. 12, no. 2, pp. 397-407, 2002.

[14] A. M. Wazwaz, "New solitary-wave special solutions with compact support for the nonlinear dispersive $K(m, n)$ equations," Chaos, Solitons and Fractals, vol. 13, no. 2, pp. 321-330, 2002.

[15] L. Zhang, L.-Q. Chen, and X. Huo, "Peakons and periodic cusp wave solutions in a generalized Camassa-Holm equation," Chaos, Solitons and Fractals, vol. 30, no. 5, pp. 1238-1249, 2006.

[16] Y. A. Li and P. J. Olver, "Convergence of solitary-wave solutions in a perturbed bi-Hamiltonian dynamical system. I. Compactons and peakons," Discrete and Continuous Dynamical Systems, vol. 3, no. 3, pp. 419-432, 1997.

[17] Y. A. Li and P. J. Olver, "Convergence of solitary-wave solutions in a perturbed bi-Hamiltonian dynamical system. II. Complex analytic behavior and convergence to non-analytic solutions," Discrete and Continuous Dynamical Systems, vol. 4, no. 1, pp. 159-191, 1998.

[18] P. Rosenau, "Nonlinear dispersion and compact structures," Physical Review Letters, vol. 73, no. 13, pp. 1737-1741, 1994.

[19] Z. Yan, "New families of solitons with compact support for Boussinesq-like $B(m, n)$ equations with fully nonlinear dispersion," Chaos, Solitons and Fractals, vol. 14, no. 8, pp. 1151-1158, 2002.

[20] Y. Zhu, "Exact special solutions with solitary patterns for Boussinesq-like $B(m, n)$ equations with fully nonlinear dispersion," Chaos, Solitons and Fractals, vol. 22, no. 1, pp. 213-220, 2004.
[21] Y. Zhu and C. Lu, "New solitary solutions with compact support for Boussinesq-like $B(2 n, 2 n)$ equations with fully nonlinear dispersion," Chaos, Solitons \& Fractals, vol. 32, no. 2, pp. 768$772,2007$.

[22] Z. Liu, Q. Lin, and Q. Li, "Integral approach to compacton solutions of Boussinesq-like $B(m, n)$ equation with fully nonlinear dispersion," Chaos, Solitons and Fractals, vol. 19, no. 5, pp. 10711081, 2004.

[23] A.-M. Wazwaz, "Nonlinear variants of the improved Boussinesq equation with compact and noncompact structures," Computers \& Mathematics with Applications, vol. 49, no. 4, pp. 565-574, 2005.

[24] S. Lai and Y. Wu, "The asymptotic solution of the Cauchy problem for a generalized Boussinesq equation," Discrete and Continuous Dynamical Systems B, vol. 3, no. 3, pp. 401-408, 2003.

[25] S. Lai, Y. H. Wu, and X. Yang, "The global solution of an initial boundary value problem for the damped Boussinesq equation," Communications on Pure and Applied Analysis, vol. 3, no. 2, pp. 319-328, 2004.

[26] A.-M. Wazwaz, "Compactons and solitary wave solutions for the Boussinesq wave equation and its generalized form," Applied Mathematics and Computation, vol. 182, no. 1, pp. 529-535, 2006.

[27] K. Weierstrass, Mathematische werke V Johnson, New York, NY, USA, 1915.

[28] E. T. Whittaker and G. N. Watson, A Course of Modern Analysis, Cambridge University Press, Cambridge, UK, 1996.

[29] K. Chandrasekharan, Elliptic Functions, Springer, Berlin, Germany, 1985.

[30] M. Abramovitz and I. A. Stegun, Handbook of Mathematical Functions, Dover, New York, NY, USA, 9th edition, 1972.

[31] S. Lai, "Different physical structures of solutions for a generalized Boussinesq wave equation," Journal of Computational and Applied Mathematics, vol. 231, no. 1, pp. 311-318, 2009. 


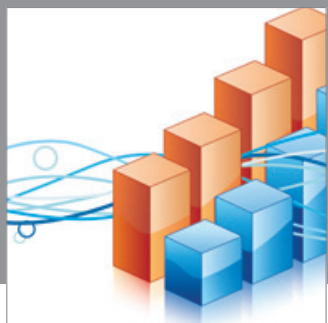

Advances in

Operations Research

mansans

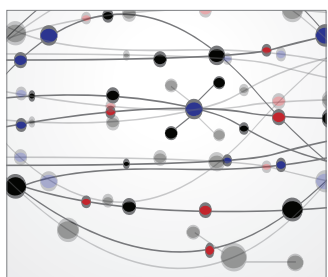

The Scientific World Journal
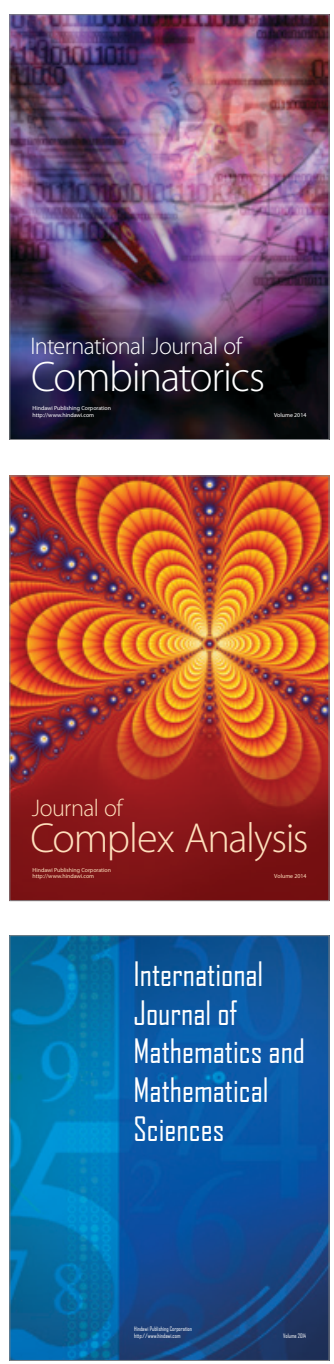
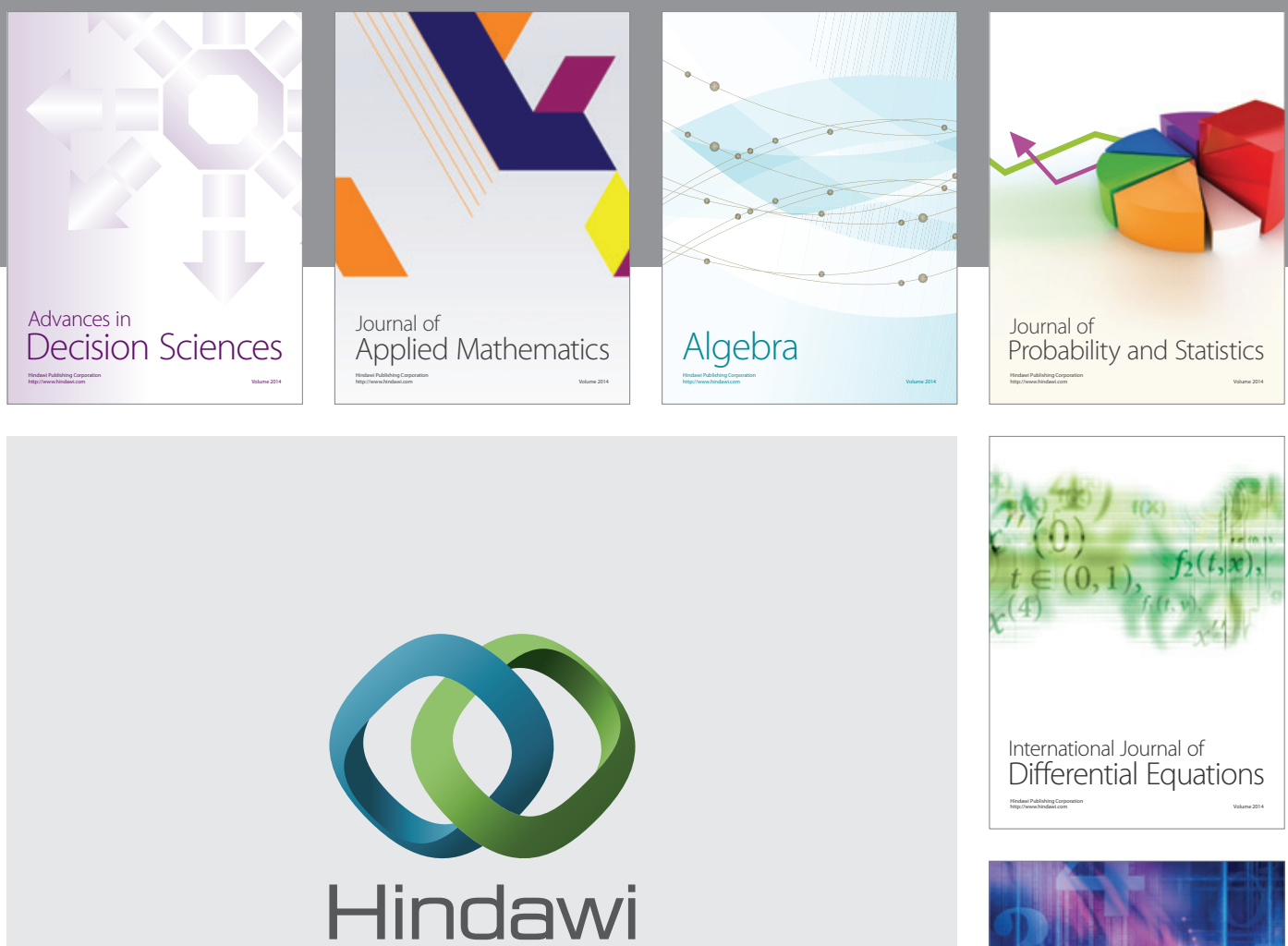

Submit your manuscripts at http://www.hindawi.com
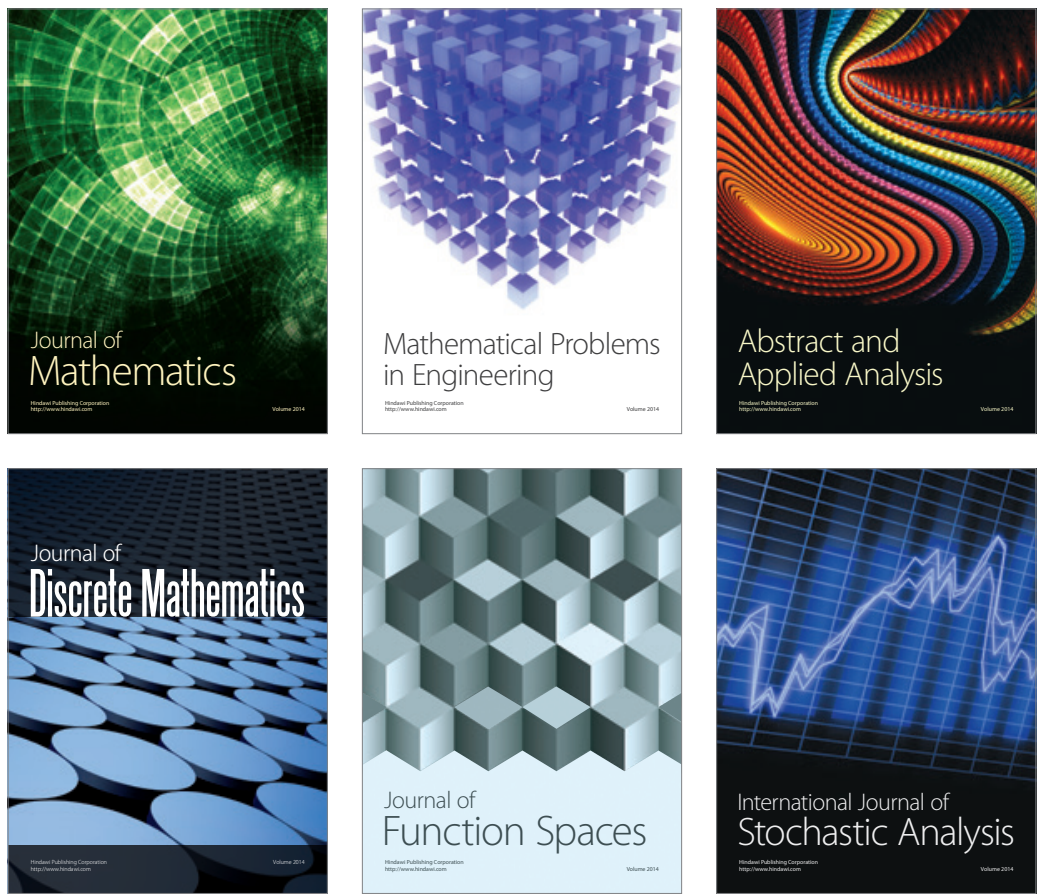

Journal of

Function Spaces

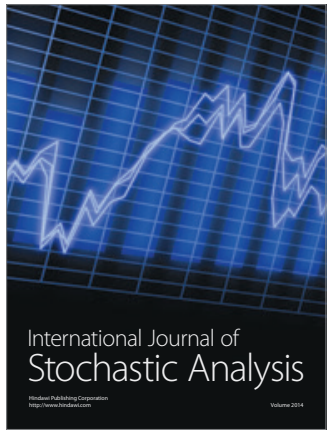

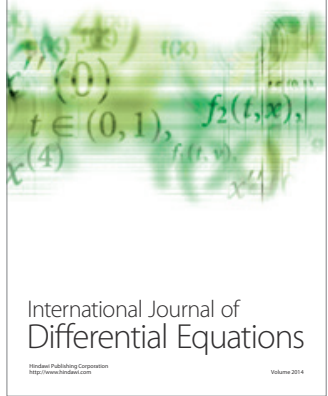
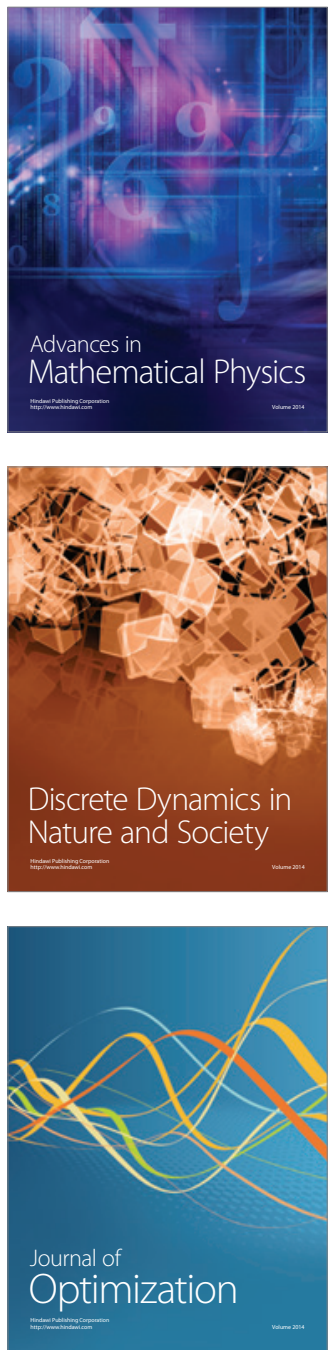